\title{
Comparación de parámetros productivos de dos líneas cárnicas de cuyes en la costa central del Perú
}

\author{
Comparison of productive parameters of two guinea pig meat lines in the \\ central coast of Peru
}

\author{
Graciela Yamada A. ${ }^{1}$, Víctor Bazán R. ${ }^{1,2}$, Nadia Fuentes N. ${ }^{1}$
}

\section{Resumen}

El objetivo del estudio fue comparar el comportamiento productivo de dos líneas cárnicas de cuyes (Cavia porcellus) criados a nivel de la costa central del Perú. Se utilizaron dos líneas genéticas de cuyes durante la etapa de crecimiento y engorde: un grupo con 20 cuyes machos, progenie de cuyes mejorados en la sierra peruana, línea cárnica $\mathrm{G}$, y otro grupo con 20 cuyes machos, mejorados en la costa peruana, línea cárnica H. Los cuyes fueron criados en jaulas individuales a partir del destete ( $15 \pm 2$ días) hasta la semana 11, recibiendo un mismo régimen alimenticio a base de maíz chala forrajera, afrecho de trigo y agua fresca. El peso al nacimiento para los cuyes $\mathrm{G}$ y $\mathrm{H}$ fue de $146.5 \pm$ 28.2 y $119.0 \pm 19.2 \mathrm{~g}$, respectivamente, el peso al destete fue de $306.5 \pm 49.1$ y $227.0 \pm 58.2$, respectivamente, y el peso a la semana 11 fue de $965.5 \pm 100.1$ y $868.0 \pm 91.6$, respectivamente $(\mathrm{p}<0.05$ en todos los casos). No se encontró diferencia significativa en ganancia diaria de peso por día, ganancia de peso total, índice de conversión alimenticia y rendimiento de carcasa; sin embargo, los cuyes de la línea $\mathrm{H}$ mostraron un mayor consumo diario de alimento y consumo total de alimento y menor peso de carcasa que los cuyes de la línea $G(p<0.05)$. Se concluye que los cuyes $G$ presentan un mayor peso al beneficio (semana 11), así como un mayor peso de canal y mejor retribución económica que los cuyes $\mathrm{H}$.

Palabras claves cuyes; parámetros productivos; líneas cárnicas; costa central

\section{Abstract}

The aim of this study was to compare the productive performance of two guinea pig meat lines (Cavia porcellus) reared in the central coast of Peru. Two genetic lines of guinea pigs were used during the growth and fattening stage: a group of 20 male guinea pig, progeny of improved guinea pigs in the Peruvian highlands, meat line G, and another

\footnotetext{
${ }^{1}$ Estación Experimental Huaral, Centro de Investigación IVITA, Facultad de Medicina Veterinaria, Universidad Nacional Mayor de San Marcos, Lima, Perú

${ }^{2}$ E-mail:vbazanr@unmsm.edu.pe
}

Recibido: 27 de abril de 2018

Aceptado para publicación: 28 de noviembre de 201 
group of 20 improved male guinea pigs in the Peruvian coast, meat line H. The guinea pigs were reared in individual cages from weaning ( $15 \pm 2$ days) until week 11 , receiving the same diet based on Zea mays var. indurata, wheat bran and fresh water. The birth weight for guinea pigs $\mathrm{G}$ and $\mathrm{H}$ was $146.5 \pm 28.2$ and $119.0 \pm 19.2 \mathrm{~g}$, respectively, the weaning weight was $306.5 \pm 49.1$ and $227.0 \pm 58.2$, respectively, and the weight at week 11 was $965.5 \pm 100.2$ and $868.0 \pm 91.6$, respectively ( $p<0.05$ in all cases). No significant difference was found in daily weight gain per day, total weight gain, feed conversion ratio and carcass yield; however, guinea pigs of line $\mathrm{H}$ showed a higher daily feed consumption and total feed consumption, and lower carcass weight than guinea pigs of line $G(p<0.05)$. It is concluded that the guinea pigs have a greater body weight at age of slaughter (week 11 ), as well as a greater carcass weight and better economic retribution than the guinea pigs $\mathrm{H}$.

Key words: guinea pig; productive parameters; meat lines; central coast

\section{INTRODUCCIÓN}

En el Perú, la crianza de cuyes (Cavia porcellus) viene presentando una mejora sustancial, gracias a los trabajos de investigación, sobre todo, para la obtención de cuyes genéticamente mejorados, que se caracterizan por su precocidad (raza Perú) y prolificidad (raza Andina) (Chauca, 2013), su alto rendimiento de carcasa y su adaptación a diferentes medioambientes. Diversas instituciones han introducido al mercado razas y líneas genéticas de cuyes con el fin de producir carne, y estos animales se han cruzado con cuyes criollos, mejorando el rendimiento de carcasa, la conversión alimenticia y los ingresos del criador. Lamentablemente, son escasos los registros de producción que permitan obtener una información real de las bondades de estos cruzamientos, generando a veces bajos resultados y deficientes niveles productivos (Meza et al., 2017). Solarte et al. (2002b) señala que aún persisten limitaciones para consolidar un programa debidamente estructurado para el mejoramiento genético en los cuyes.

La Universidad Nacional Mayor de San Marcos (UNMSM) a través de la Estación Experimental de El Mantaro del Centro de Investigación IVITA ha logrado desarrollar los cuyes G; una línea cárnica mejorada a nivel de sierra. La estación Huaral del IVITA, ubicada en el departamento de Lima, inicia la crianza de estos cuyes de línea cárnica en marzo de 2016, siendo uno de los objetivos comparar las características de los cuyes mejorados a nivel de sierra frente a los cuyes mejorados desarrollado y criados a nivel del mar.

\section{Materiales y Métodos}

El presente trabajo se realizó en el módulo demostrativo de cuyes de la Estación IVITA Huaral de la Facultad de Medicina Veterinaria (FMV) de la Universidad Nacional Mayor de San Marcos (UNMSM), ubicado en el distrito de Huaral, región Lima, Perú. La estación se encuentra a una altura de $200 \mathrm{msnm}$, con temperatura promedio de $19.4{ }^{\circ} \mathrm{C}, 16 \mathrm{~mm}$ de precipitación pluvial media anual y una humedad relativa de $82.3 \%$.

Se emplearon 40 cuyes machos, 20 cuyes de la línea cárnica G y 20 cuyes de la línea cárnica $\mathrm{H}$ (raza mejorada y criada en Huaral) de diferentes jaulas, destetadas el mismo día y seleccionadas al azar. Todos los animales nacieron en el módulo de cuyes de la Estación IVITA Huaral y fueron destetados a $\operatorname{los} 15 \pm 2$ días de edad. Los animales se colocaron en jaulas individuales de 0.40 x 0.50 $\mathrm{m}$, a una altura de $0.50 \mathrm{~m}$, desde el destete hasta las 11 semanas de edad. 
Para la alimentación se empleó afrecho de trigo (18\% proteína y $13 \%$ fibra cruda) y maíz chala forrajera Zea mays $\mathrm{L}$ variedad Marginal T-28 (9\% proteína y $28 \%$ de fibra cruda) y agua. Cada animal fue alimentado con $30 \mathrm{~g}$ de afrecho y de $200 \mathrm{~g}$ de chala forrajera en las primeras tres semanas, mientras que en las ocho semanas restantes recibieron $30 \mathrm{~g}$ de afrecho y $300 \mathrm{~g}$ de chala forrajera. El agua se ofreció a los animales en horas de la mañana en pocillos de arcilla de $500 \mathrm{ml}$ de capacidad. La chala ofrecida era fresca con presencia de mazorcas con granos en estado lechoso de aproximadamente 3.5 meses. El corte se realizaba el día anterior al suministro, obtenido de los cultivos de la Estación IVITA Huaral.

Los animales fueron pesados semanalmente en forma individual al nacimiento $(\mathrm{PN})$, $y$ en el destete (PD) a las 08:00, antes del suministro del alimento. Al cumplir las 11 semanas fueron pesados antes y después del faenado, obteniendo el peso de la carcasa (PC) después del oreo. Para el beneficio, se consideró un periodo de ayuno de 12 horas. El peso de la carcasa incluye piel, cabeza, extremidades y vísceras (corazón, pulmones, hígado y riñones). Para el pesaje se empleó una balanza digital con $\pm 10 \mathrm{~g}$ de sensibilidad.

El índice de consumo de alimento (ICA) se obtuvo por individuo, sumando el consumo de materia seca del afrecho y de la chala forrajera ofrecido en horas de la mañana, y luego restando la materia seca del afrecho y la chala forrajera sobrante recolectados en bandejas estercoleras, descartando las heces y orina. La materia seca del afrecho y de la chala forrajera fueron analizados en el laboratorio de Bioquímica, Nutrición y Alimentación Animal de la FMV-UNMSM al inicio del estudio, obteniéndose $85 \%$ de materia seca para el afrecho y $21.8 \%$ para la chala forrajera. Para el pesaje del alimento ofrecido y sobrante se empleó una balanza digital con capacidad de $200 \mathrm{~g}$ con una precisión de $\pm 2 \mathrm{~g}$. La ganancia de peso diaria (GPD), el consumo diario de alimento (CDA) y el índice de conversión alimenticia (ICA) fueron analizados en tres etapas: del destete hasta las 6 semanas, de la semana 6 a la 11, y entre el destete y la semana 11. La retribución económica (RE) se determinó mediante la diferencia entre el precio de la carcasa en el mercado y el costo de la ración por animal.

Se empleó el análisis de varianza simple para comparar el peso de los machos $\mathrm{G}$ y $\mathrm{H}$ con un nivel de significancia de $\mathrm{p}<0.05$. Se utilizó el paquete estadístico Statgraphics Centurion v. 17.2.04.

\section{Resultados y Discusión}

\section{Peso y Ganancia de Peso}

Los cuyes $\mathrm{G}$ nacieron con mayor peso que los cuyes $H(146.5 \pm 28.2$ vs. $119.0 \pm$ $19.2 \mathrm{~g})$ ( $\mathrm{p}<0.05$; Cuadro 1). Estos pesos fueron similares a los obtenidos en cuyes Cieneguilla $146.9 \pm 33.5 \mathrm{~g}$ por Rodríguez et al. (2013).

El peso al destete de los cuyes $\mathrm{G}$ fue de $306.5 \pm 49.2$, logrando un incremento de peso del $115.6 \%$ en los $15 \pm 2$ días de lactancia, mientras que los cuyes $\mathrm{H}$ pesaron $227.0 \pm$ $58.2 \mathrm{~g}$, lo que representa un incremento de $91.56 \%$ en el mismo periodo de lactancia (Cuadro 1). Rodríguez et al. (2013) reporta $310.9 \pm 96.3 \mathrm{~g}$ de peso al destete (14.2 días), con un incremento de $111.6 \%$, mientras que Solarte et al. (2002a) reportó pesos de $276 \mathrm{~g}$ en cuyes destetados en Colombia con una ganancia del 106\%. Los pesos obtenidos por los cuyes $\mathrm{G}$ al destete fueron mayores a los 288 g obtenidos por Jiménez (2005) en cuyes de línea mejorada y a los $260 \pm 57 \mathrm{~g}$ reportados por Chauca et al. (2013) en cuyes de una línea sintética del Instituto Nacional de Innovación Agraria (INIA) destetados a los 14 días, donde los incrementos de peso desde el nacimiento al destete fueron de $89.5 \mathrm{y}$ $99.0 \%$, respectivamente.

El peso a las 11 semanas fue similar para ambos genotipos $(965.50 \pm 100.13 \mathrm{~g}$ para los cuyes $\mathrm{G}$ y $868.00 \pm 91.57 \mathrm{~g}$ para los cuyes $\mathrm{H}$ ). 
Cuadro 1. Comparación productiva entre dos líneas cárnicas de cuyes en la costa central del Perú

\begin{tabular}{lcc}
\hline & Línea G & Línea H \\
\hline Peso al nacimiento $(\mathrm{g})$ & $146.5 \pm 28.2^{\mathrm{a}}$ & $119.0 \pm 19.2^{\mathrm{b}}$ \\
Peso al destete $(\mathrm{g})$ & $306.5 \pm 49.1^{\mathrm{a}}$ & $227.0 \pm 58.2^{\mathrm{b}}$ \\
Peso a las 11 semanas $(\mathrm{g})$ & $965.5 \pm 100.1^{\mathrm{a}}$ & $868.0 \pm 91.6^{\mathrm{b}}$ \\
Ganancia total de peso (g) & $659.0 \pm 84.9^{\mathrm{a}}$ & $641.0 \pm 55.8^{\mathrm{a}}$ \\
Ganancia diaria de peso (g/cuy/día) & $10.5 \pm 1.4^{\mathrm{a}}$ & $10.2 \pm 0.9^{\mathrm{a}}$ \\
Consumo total (g MS/cuy) & $3500 \pm 41^{\mathrm{a}}$ & $3535 \pm 58^{\mathrm{b}}$ \\
Consumo diario (g MS/cuy/día) & $55.6 \pm 0.6^{\mathrm{a}}$ & $56.1 \pm 0.9^{\mathrm{a}}$ \\
Índice de conversión alimenticia & $5.40 \pm 0.71^{\mathrm{a}}$ & $5.55 \pm 0.48^{\mathrm{a}}$ \\
\hline
\end{tabular}

${ }^{a b}$ Letras diferentes dentro de filas indican diferencias estadísticas significativas $(p<0.05)$

Estos pesos están dentro del rango ofrecido en el mercado (Morales et al., 2011). Las ganancias de peso fueron similares entre ambos genotipos, registrándose una ganancia diaria de peso promedio de $10.5 \pm 1.4 \mathrm{~g}$ para los cuyes $\mathrm{G}$ y de $10.2 \pm 0.9 \mathrm{~g}$ para los cuyes $\mathrm{H}$. Estos resultados fueron similares a los 10.3-11.1 g/cuy/día obtenidos por Sánchez et al. (2013) al comparar tipos de comederos en cuyes del Centro de Investigación IVITA El Mantaro, pero mayores a los 8.6$9.3 \mathrm{~g} /$ cuy/día reportados por Mamani et al. (2015) en cuyes sometidos al pastoreo en dicho centro experimental.

\section{Consumo de Materia Seca}

El consumo diario de materia seca fue similar entre las dos líneas genéticas, en tanto que el consumo total de materia seca presentó diferencia significativa entre las dos líneas de cuyes ( $<<0.05$; Cuadro 1$)$. Valores similares fueron reportados por Mattos et al. (2003) y Torres et al. (2013) en cuyes de la línea Perú y en cuyes mejorados de la línea cárnica de la Estación IVITA El Mantaro (59.9 g MS/cuy/día). Al evaluar el consumo de materia seca por periodos (Cuadro 2), los cuyes G consumieron $49.2 \mathrm{~g} \mathrm{MS} /$ cuy/día entre el destete y la semana 6 , mientras que los cuyes $\mathrm{H}$ consumieron $49.6 \mathrm{~g} \mathrm{MS/cuy/día,} \mathrm{no}$ habiendo diferencia significativa. No obstante, entre la semana 6 y la semana 11 y entre el destete y la semana 11 se presentó un mayor consumo en los cuyes $\mathrm{H}(\mathrm{p}<0.05)$ (Cuadro 2). Mamani et al. (2015) obtuvieron, por otro lado, en cuyes al pastoreo en la Estación IVITA El Mantaro, consumos mayores de materia seca (74.8-90.3 g MS/cuy/día).

Índice de Conversión Alimenticia (ICA)

No se encontró diferencias significativas en la conversión alimenticia entre grupos en ninguno de los tres periodos bajo evaluación (Cuadro 2). Mattos et al. (2003) obtuvo conversiones alimenticias similares de $5.4 \mathrm{y}$ 6.4 en cuyes sometidos a un suplemento de ensilado de pescado y control, respectivamente. Así mismo, Torres et al. (2013) utilizando cuyes sometidos a una cepa probiótica obtuvo valores de ICA entre 4.33 y 5.04. Por otro lado, Mamani et al. (2015) reportaron valores de 8.4 a 10.7 en cuyes sometidos a diferentes periodos de descanso en el pastoreo de pasturas asociadas. 
Cuadro 2. Ganancia diaria de peso (g), consumo de materia seca (g/día) y conversión alimenticia en dos líneas cárnicas de cuyes en la costa central del Perú

\begin{tabular}{ccc}
\hline Periodo & Cuyes G & Cuyes H \\
\hline Ganancia de peso diario (g) & & \\
15 días - 6 semanas & $9.9^{\mathrm{a}}$ & $8.8^{\mathrm{a}}$ \\
6 - 11 semanas & $10.9^{\mathrm{a}}$ & $11.3^{\mathrm{a}}$ \\
15 días - 11 semanas & $10.5^{\mathrm{a}}$ & $10.2^{\mathrm{a}}$ \\
Consumo de materia seca/cuy/día (g) & & \\
15 días - 6 semanas & $49.2^{\mathrm{a}}$ & $49.6^{\mathrm{a}}$ \\
6 - 11 semanas & $60.7^{\mathrm{a}}$ & $61.4^{\mathrm{b}}$ \\
15 días - 11 semanas & $55.6^{\mathrm{a}}$ & $56.1^{\mathrm{b}}$ \\
Conversión alimenticia & & \\
15 días - 6 semanas & $5.28^{\mathrm{a}}$ & $5.89^{\mathrm{a}}$ \\
11 días - 11 semanas & $5.80^{\mathrm{a}}$ & $5.53^{\mathrm{a}}$ \\
\end{tabular}

\section{Peso y Rendimiento de Carcasa}

Los cuyes $\mathrm{G}$ presentaron un mayor peso de carcasa frente a los cuyes $\mathrm{H}(\mathrm{p}<0.05$, Cuadro 3). Los pesos de las carcasas fueron mayores a $\operatorname{los} 420 \pm 54 \mathrm{~g}$ en cuyes de la raza Perú reportados en México por Xicohtencatl et al. (2013), pero fueron menores a los reportados por Chauca (2016) en cuyes de la raza Perú $(752.4 \pm 126.1 \mathrm{~g})$ a las 9 semanas. Así mismo, Mattos et al., 2003 en cuyes alimentados con forraje y diferentes niveles de ensilado de pescado en el concentrado obtuvieron entre 457 y $684 \mathrm{~g}$ de carcasa en animales sacrificados a las 10 semanas.

El rendimiento de carcasa fue similar entre los genotipos $\mathrm{G}$ y H (72.7 y $72.3 \%$, respectivamente). Estos rendimientos de carcasa fueron similares a los reportados por Mattos et al. (2003) de 68.7-72.6\% y por Huamaní et al. (2016) de 69.8 a $73.7 \%$ en cuyes sometidos a tres sistemas de alimentación. Rendimientos menores fueron los obtenidos por Xicohtencatl et al. (2013) de 44\% y por Chauca (2016) de 54.4 y $67.4 \%$ para cuyes
Cuadro 3. Peso y rendimiento de las carcasas de cuyes $\mathrm{G}$ y $\mathrm{H}$ criados a nivel del mar

\begin{tabular}{lcc}
\hline Índices & Cuyes G & Cuyes H \\
\hline $\begin{array}{c}\text { Peso vivo } \\
(\mathrm{g})\end{array}$ & $966 \pm 100^{\mathrm{a}}$ & $868 \pm 92^{\mathrm{b}}$ \\
$\begin{array}{c}\text { Peso de la } \\
\text { canal (g) }\end{array}$ & $702 \pm 82^{\mathrm{a}}$ & $628 \pm 66^{\mathrm{b}}$ \\
$\begin{array}{c}\text { Rendimiento } \\
(\%)\end{array}$ & $72.7 \pm 2.4^{\mathrm{a}}$ & $72.3 \pm 1.7^{\mathrm{a}}$ \\
\hline
\end{tabular}

ab Letras diferentes dentro de filas indican diferencias estadísticas significativas $(p<0.05)$

alimentados con forrajes y concentrados, respectivamente.

La retribución económica fue mejor en los cuyes $G$ frente a los cuyes $H(p<0.05$; Cuadro 4), debido a que los cuyes $G$ mostraron una mayor ganancia de peso. 
Cuadro 4. Retribución económica en cuyes $\mathrm{G}$ y $\mathrm{H}$ criados a nivel del mar

\begin{tabular}{lcc}
\hline Índices & Cuyes $\mathrm{G}$ & Cuyes $\mathrm{H}$ \\
\hline Peso final de la carcasa $(\mathrm{kg})$ & $0.702^{\mathrm{a}}$ & $0.628^{\mathrm{b}}$ \\
Precio de la carcasa $(\mathrm{S} / . / \mathrm{kg})$ & 23.00 & 23.00 \\
Ingreso bruto por cuy vendido (S/.) & $16.15^{\mathrm{a}}$ & $14.43^{\mathrm{b}}$ \\
Alimentación & & \\
$\quad$ Concentrado & 1.312 & 1.305 \\
Consumo $(\mathrm{kg})$ & 0.77 & 0.77 \\
Precio $(\mathrm{S} / . / \mathrm{kg})$ & 1.01 & 1.00 \\
Total & & \\
Forraje & 9.953 & 10.144 \\
Consumo $(\mathrm{kg})$ & 0.06 & 0.06 \\
Precio $(\mathrm{S} / . / \mathrm{kg})$ & 0.60 & 0.61 \\
Total & 1.61 & 1.61 \\
Costo de alimentación $(\mathrm{S} /)$. & $14.54^{\mathrm{a}}$ & $12.82^{\mathrm{b}}$ \\
Retribución económica por cuy logrado $(\mathrm{S} /)$. & 100.0 & 88.2 \\
Retribución económica relativa $(\%)$ &
\end{tabular}

${ }^{a b}$ Letras diferentes dentro de filas indican diferencias estadísticas significativas $(p<0.05)$

\section{Conclusiones}

- Cuyes alimentados con afrecho de trigo y chala forrajera de la línea cárnica $\mathrm{G}$ presentan una mayor ganancia de peso entre el destete y la semana 11 en comparación a los cuyes de línea cárnica $\mathrm{H}$.

- El peso de carcasa fue mayor en los cuyes de la línea cárnica $\mathrm{G}$ en comparación con los cuyes de la línea cárnica $\mathrm{H}$.

- Los cuyes de línea cárnica G presentan mejor retribución económica frente a los cuyes de línea cárnica $\mathrm{H}$.

\section{Literatura Citada}

1. Chauca L. 2013. Crianza del cuy $\mathrm{Ca}$ via porcellus y su impacto en el desarrollo rural. En: XXXVI Reunión Científica Anual de la Asociación Peruana de Producción Animal. Lima, Perú.
2. Chauca L. 2016. Impactos y retos en la investigación en cuyes para su desarrollo y consolidación empresarial. En: Simposio Nacional «Avances y Perspectivas en la Producción de cuyes». Lima. Perú: UNALM.

3. Chauca L, Huaman M, Reynaga M, Muscari J, Higaonna R. 2013. Comportamiento reproductivo de cuyes de la línea sintética (INIA P 5/8 IxA3/8). En: XXXVI Reunión Científica Anual de la Asociación Peruana de Producción Animal. Lima. Perú.

4. Huamaní G, Zea O, Gutierrez G, Vilchez C. 2016. Efecto de tres sistemas de alimentación sobre el comportamiento productivo y perfil de ácidos grasos de carcasa de cuyes (Cavia porcellus). Rev Inv Vet Peru 27: 486494. doi: 10.15381/rivep.v27i3.12004

5. Jiménez A. 2005. Determinación de parámetros productivos y reproductivos de cuyes mejorados con sistemas de crianza en jaula y en poza. Tesis de In- 
geniero Zootecnista. Riobamba Ecuador: Escuela Superior Politécnico de Chimborazo. $115 \mathrm{p}$.

6. Mamani R, Jiménez R, San Martín F, Huamán H, Ara M, Carcelén F, Huamán A. 2015. Determinación del periodo óptimo de descanso de la pastura asociada Lolium multiflorum, trifulium pratense y Medicago sativa, pastoreada por cuyes en la sierra Central del Perú. Rev Inv Vet Peru 26: 404411. doi: 10.15381/rivep.v26i3.11174

7. Mattos J, Chauca L, San Martín F, Carcelén F. Arbaiza T. 2003. Uso del ensilado biológico de pescado en la alimentación de cuyes mejorados. Rev Inv Vet Peru 14: 89-96. doi: 10.15381/ rivep.v14i2.1612

8. Meza E, Raymondi J, Cisneros S. 2017. Evaluación genética de un plantel de cuyes reproductores de genotipo Perú. Rev Inv Vet Peru 28: 293-298. doi: 10.15381/rivep.v28i2.13067

9. Morales A, Carcelén F, Ara M, Arbaiza T, Chauca L. 2011. Evaluación de dos niveles de energía en el comportamiento productivo de cuyes (Cavia porcellus) de la raza Perú. Rev Inv Vet Peru 22: 177-182. doi: 10.15381/ rivep.v22i3.254

10. Rodríguez H, Palomino M, Hidalgo V, Gutiérrez G. 2013. Efectos de factores fijos y al azar sobre el peso al na- cimiento y al destete en cuyes de la costa central del Perú. Rev Inv Vet Peru 24: 16-24. doi: 10.15381/rivep.v24i1.1647

11. Sánchez R, Jiménez R, Huamán H, Bustamante J, Huamán A. 2013. Respuesta reproductiva y económica al uso de cuatro tipos de comederos para forraje en la crianza de cuyes. Rev Inv Vet Peru 24: 441-450. doi: 10.15381/ rivep.v24i4.2733

12. Solarte C, Imuez A, Pérez T. $2002 a$. Modelo animal multicarácter para la estimación de parámetros genéticos del Cavia porcellus en Colombia. Cuban J Agr Sci 36: 19-24.

13. Solarte C, Soto F, Pérez T. 2002b. Modelo animal multicarácter para la selección de reproductores Cavia porcellus en Colombia. Cuban J Agr Sci 36: 25-29.

14. Torres C, Carcelén F, Ara M, San Martín F, Jiménez $R$, Quevedo $W$, Rodríguez J. 2013. Efecto de la suplementación de una cepa probiótica sobre los parámetros productivos del cuy. Rev Inv Vet Peru 24: 433-440. doi: 10.15381/rivep.v24i4.2729

15. Xicohtencatl P, Barrera S, Orozco T, Torres F, Monsivais R. 2013. Parámetros productivos de cuyes $(\mathrm{Ca}$ via porcellus) del nacimiento al sacrificio en Nayarit, México. Abanico Vet 3: 36-43. 